\title{
Pulmonary artery distensibility in pulmonary arterial hypertension: an MRI pilot study
}

\author{
C. Jardim*, C.E. Rochitte ${ }^{\#}$, M. Humbert ${ }^{\mp}$, G. Rubenfeld ${ }^{+}$, D. Jasinowodolinski ${ }^{\#}$, \\ C.R.R. Carvalho* and R. Souza*,\#,
}

ABSTRACT: Pulmonary arterial hypertension (PAH) is a disease of the small vessels in which there is a substantial increase in pulmonary vascular resistance leading to right ventricle failure and death. Invasive haemodynamic evaluation is mandatory not only for diagnosis confirmation but also to address prognosis and eligibility for the use of calcium-channel blockers through an acute vasodilator challenge. Noninvasive surrogate response markers to the acute vasodilator test have been sought.

In the present study, the relationship between pulmonary artery distensibility, assessed using magnetic resonance imaging (MRI), and response to acute vasodilator tests was investigated. In total, 19 patients diagnosed with idiopathic PAH without any specific treatment were evaluated. Within a 48-h window after pulmonary artery catheterisation, patients underwent cardiac MRI.

Cardiac index, calculated after the determination (invasively and noninvasively) of cardiac output, showed excellent correlation, as did right atrial pressure and right ventricle ejection fraction. Pulmonary artery distensibility was significantly higher in responders. A receiver operating characteristic curve analysis has shown that $10 \%$ distensibility was able to differentiate responders from nonresponders with $100 \%$ sensitivity and $56 \%$ specificity.

The present findings suggest that magnetic resonance imaging and pulmonary artery distensibility may be useful noninvasive tools for the evaluation of patients with pulmonary hypertension.

KEYWORDS: Acute vasodilator response, haemodynamic evaluation, magnetic resonance imaging, pulmonary artery distensibility, pulmonary hypertension

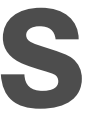
ince the beginning of the 20th century, there have been substantial contributions to the understanding of pulmonary circulation [1]. The development of right heart catheterisation (RHC) has allowed the recognition of pulmonary circulation as a low-resistance and high-compliance system [2]. Pulmonary hypertension is defined by a mean pulmonary artery pressure $\left(\bar{P}_{\mathrm{pa}}\right)>25 \mathrm{mmHg}$ at rest or $>30 \mathrm{mmHg}$ in exercise $[3,4]$. There is a multitude of conditions in which pulmonary hypertension develops and great efforts have been dedicated to the adequate classification of these diseases [5]. When no associated condition can be identified, the diagnosis of idiopathic pulmonary arterial hypertension (IPAH) is established. IPAH is a rare condition with high mortality rates even after the development of different treatment strategies in recent years, including the use of prostanoids, endothelin receptor antagonists, calcium-channel blockers (CCBs) and phosphodiesterase inhibitors [6].

A small proportion of IPAH patients present sustained response to CCBs. For the identification of these patients, it is necessary to evaluate acute vasodilator response [7, 8]. During RHC, patients are challenged with vasodilators and, if a nearnormalisation of $\bar{P}_{\text {pa }}$ and pulmonary vascular resistance (PVR) occurs, the patient is classified as an acute responder. The importance of assessing responsiveness is based primarily on eligibility for CCB therapy $[9,10]$. However, response to acute vasodilator testing may also correlate with better preserved cardiac function, thus making it a possible surrogate for vascular remodelling [11].

Great efforts have been dedicated to the search of noninvasive markers that reflect not only haemodynamic parameters but also acute responsiveness to vasodilators [12]. Imaging techniques, such as echocardiography, computed tomography (CT) and magnetic resonance imaging (MRI), may also help in the assessment of IPAH patients not only with anatomical but also with functional data [13]. It is known that echocardiography is useful for the estimation of systolic pulmonary artery pressure and $\bar{P}_{\text {pa }}$ but it lacks reliability for
AFFILIATIONS

*Pulmonary Division, Pulmonary Hypertension Unit, Heart Institute, University of São Paulo Medical School, and

\#Fleury Research Institute, São Paulo, Brazil.

"National Pulmonary Hypertension Reference Centre, Hôpital Antoine Béclère, Paris-Sud University, Clamart, France.

+Pulmonary and Critical Care Division, University of Washington, Seattle, WA, USA.

CORRESPONDENCE

R. Souza

R. Afonso de Freitas 451 Ap 112

São Paulo

04006-052

Brazil

Fax: 551130697202

E-mail: rgrsz@uol.com.br

Received:

February 042006

Accepted after revision:

November 152006

SUPPORT STATEMENT

The present study was funded by an educational grant received from Coordenação de Aperfeiçoamento de Pessoal de Nivel Superior, Ministry of Education, Brasilia, Brazil.

STATEMENT OF INTEREST None declared.

Online ISSN 1399-3003 
the measurement of right atrial pressure (RAP) and is unable to accurately estimate the cardiac index (CI) [14]. Chest CT, especially if performed with intravenous contrast, may provide data on right ventricular performance and predict early death in acute pulmonary embolism [15]. Moreover, MRI may be used to estimate $\bar{P}$ pa, PVR, ventricular volumes and cardiac output, thus allowing calculation of CI [16-18].

In other studies, mean pulmonary artery distensibility (mPAD) evaluated using MRI has been addressed in order to assess pulmonary haemodynamics and diagnose pulmonary vascular disease in specific settings $[19,20]$. It is believed that the mPAD may reflect the degree of vascular remodelling, thus making it a very interesting marker for evaluation of IPAH patients [21]. However, one aspect of mPAD that has not been evaluated to date is its relationship with the acute vasodilator test response.

The main objective of the present study was to investigate whether mPAD, assessed using MRI, is a noninvasive marker of acute vasodilator responsiveness in IPAH patients. The present authors also evaluated whether MRI could reflect or substitute for some of the haemodynamic parameters obtained through RHC.

\section{MATERIAL AND METHODS Study subjects}

The present authors prospectively evaluated haemodynamic measurements and MRI data from 20 consecutive patients diagnosed with IPAH between January and December 2004. The present sample size was based on an expected prevalence of responders of $\sim 15 \%$ [8] and on an SD and effect size for mPAD of 4 and $7 \%$, respectively [21]. Pulmonary arterial hypertension (PAH) was defined by $\bar{P}_{\text {pa }}>25 \mathrm{mmHg}$ and a normal pulmonary artery occlusion pressure $(P$ pao $)<15 \mathrm{mmHg}$ at rest. Secondary causes were excluded on the basis of clinical examination, laboratory studies, chest radiography, CT, echocardiography and pulmonary angiography, where necessary [5]. All patients gave written informed consent and the study was approved by the local ethics committee. One patient was excluded from the study owing to inadequate image quality at MRI.

\section{Haemodynamic measurements}

All patients underwent RHC with a triple-lumen balloontipped catheter through the internal jugular vein (Swan-Ganz, model 131F7; Baxter Edwards Critical Care Division, Irvine, CA, USA). Haemodynamic data were collected at baseline and after administration of inhaled nitric oxide (NO; $20 \mathrm{ppm}$ for $10 \mathrm{~min}$ ) [10].

The following haemodynamic variables were recorded: RAP, cardiac output, $P$ pao, $\bar{P}_{\text {pa }}$ and PVR. CI and indexed PVR (PVRi) were obtained after adjustment to body surface area (BSA).

Patients were classified as responders or nonresponders according to a decrease in $\bar{P}_{\text {pa }}$ of $\geqslant 10 \mathrm{mmHg}$ to a level $<40 \mathrm{mmHg}$ with a maintained or increased CI [8].

\section{MRI analysis}

MRI was performed within $48 \mathrm{~h}$ after RHC, without any change in clinical status or treatment. All patients underwent an MRI study in a $1.5 \mathrm{~T}$ Signa Horizon EchoSpeed (GE Healthcare, Milwaukee, WI, USA). The protocol included cine-MRI images using an ECG-gated steady-state free precession gradient-echo pulse sequence (SSFP) that allowed for dynamic evaluation of cardiovascular morphology and function. The parameters of this sequence were as follows: repetition time $3.6 \mathrm{~ms}$; echo time $1.6 \mathrm{~ms} ; 45^{\circ}$ flip angle; receiver bandwidth of $\pm 125 \mathrm{kHz}$; 34-36-cm field of view; 256 $\times 160$ matrix; 20-30 cardiac phases and 8-14 views per segment, depending on cardiac frequency in order to allow temporal resolution of $40 \mathrm{~ms}$. This sequence provided images with intense bright blood and high definition of vascular and cardiac structures. Shortaxis images (8.0-mm slice thickness, $2.0-\mathrm{mm}$ gap) from the apex to the base of the heart and a set of parallel fourchamber long-axis views (6.0-mm slice thickness, no gap) from the right ventricle (RV) inferior wall to the RV outflow tract (RVOT) were also acquired. Additionally, a long-axis RVOT view and left ventricle outflow tract views were also acquired. From those two planes, a set of 2-3 transverse short-axis views (perpendicular to the long axis) of the aortic root and main pulmonary artery were imaged using SSFP. A gradient echo with phase-contrast reconstruction was also performed in order to assess blood flow velocities. The SSFP images were used to measure the maximal and minimal sectional areas during the cardiac cycle, from which the mPAD was calculated as follows:

$$
\mathrm{mPAD}=(\text { systolic area-diastolic area }) / \text { systolic area }
$$

and expressed in \% variation (fig. 1).

Measurements of left and right ventricular volumes, ejection fraction (EF) and mass were performed using the cine-MRI short-axis views and MASS plus software analysis package (MEDIS, Leiden, the Netherlands). The calculations used by this software are based on the true Simpson's rule for volume measurements. Measurements of the flow velocities through the aorta were performed using CV-Flow (MEDIS). Both software packages were part of the Advantage Windows Workstation 4.0 (GE Healthcare). MRI was performed without supplemental $\mathrm{NO}$ as the intention was to assess patients in their basal condition.

Two independent observers blinded to the results of the haemodynamic measurements performed all image analyses. CI was obtained after adjustment of cardiac output to BSA.

\section{Statistical analysis}

Variables are presented as mean \pm SD. Comparison of the continuous variables between groups was conducted using Mann-Whitney U-tests. The Bland-Altman method was used to evaluate interobserver agreement, as well as the comparison between the CI assessed using MRI (CIMRI) and the CI assessed using RHC (CIRHC). The correlation of the haemodynamic variables and the MRI measurements was assessed through Pearson's correlation coefficient. In order to evaluate the capability of mPAD for differentiating responders and nonresponders, a receiver operating characteristic (ROC) curve was used, which was generated by plotting sensitivity against 1-specificity for all possible cut-off values.

\section{RESULTS}

Baseline haemodynamic and clinical characteristics of the 19 IPAH patients enrolled in the study are listed in table 1 . The haemodynamic variation during $\mathrm{NO}$ inhalation is summarised 

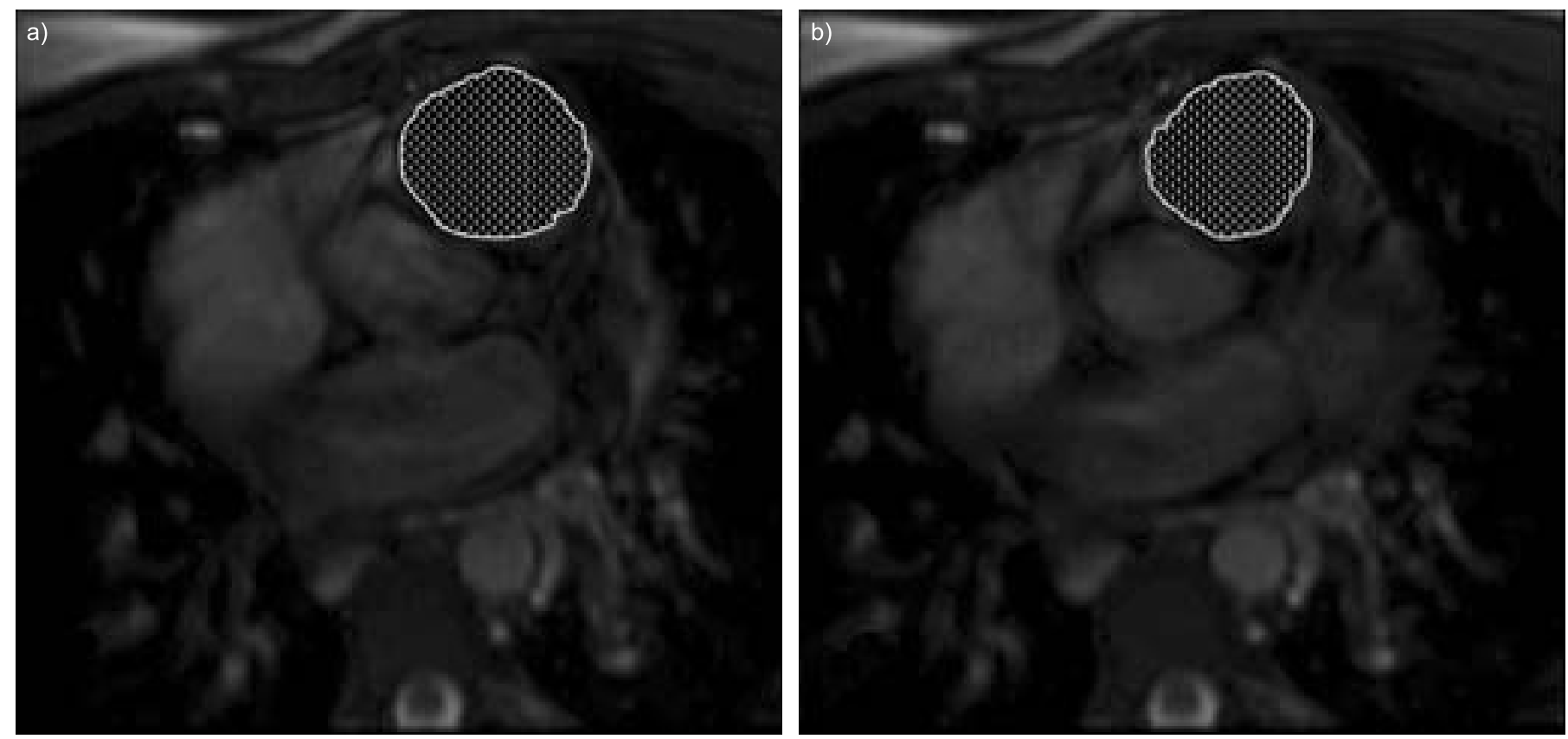

FIGURE 1. Cross-sectional image of pulmonary artery used for determination of mean pulmonary artery distensibility (mPAD) according to the following formula: $\mathrm{mPAD}=($ systolic area $(\mathrm{SA})$-diastolic area (DA)/SA. a) The marked area represents SA. b) The marked area represents DA.

in table 2. Four patients $(21 \%)$ were identified as responders after acute challenge with $\mathrm{NO}$ inhalation.

Functional and haemodynamic data obtained using MRI image analyses are shown in table 3 . The present data are consistent with previous reports of patients with chronic cor pulmonale, concerning diminished RV function and elevated RV mass and end diastolic volume with normal left ventricular function $[22,23]$.

The interobserver agreement for the CI estimation, assessed according to the Bland-Altman method, showed an error of

\begin{tabular}{|c|c|c|}
\hline TABLE 1 & \multicolumn{2}{|c|}{$\begin{array}{l}\text { Baseline clinical and haemodynamic } \\
\text { characteristics }\end{array}$} \\
\hline \multicolumn{2}{|l|}{ Subjects n } & 19 \\
\hline \multicolumn{2}{|c|}{ Age yrs } & $38 \pm 11$ \\
\hline \multicolumn{2}{|c|}{ Sex male/female $\mathbf{n}$} & $6 / 13$ \\
\hline \multicolumn{2}{|c|}{ NYHA functional class I-II/III-IV } & $5 / 14$ \\
\hline \multicolumn{2}{|c|}{$\bar{P}$ pa $\mathbf{m m H g}$} & $69 \pm 21$ \\
\hline \multicolumn{2}{|c|}{ PVRi dyn $\cdot \mathbf{s} \cdot \mathrm{cm}^{-5} \cdot \mathrm{m}^{-2}$} & $2312 \pm 1635$ \\
\hline \multicolumn{2}{|c|}{ CI L. $\min ^{-1} \cdot \mathrm{m}^{-2}$} & $2.39 \pm 1.06$ \\
\hline \multicolumn{2}{|c|}{ Ppao mmHg } & $9 \pm 5$ \\
\hline \multicolumn{2}{|l|}{ RAP $\mathrm{mmHg}$} & $12 \pm 6$ \\
\hline \multicolumn{2}{|c|}{ BSA $m^{2}$} & $1.80 \pm 0.17$ \\
\hline \multicolumn{2}{|c|}{ Cardiac frequency beats $\min ^{-1}$} & $84 \pm 9$ \\
\hline
\end{tabular}

Data are presented as mean $\pm S D$, unless otherwise stated. NYHA: New York Heart Association; $\bar{P}$ pa: mean pulmonary artery pressure; PVRi: indexed pulmonary vascular resistance; $\mathrm{Cl}$ : cardiac index; Ppao: pulmonary artery occlusion pressure; RAP: right atrial pressure; BSA: body surface area.
$0.004 \pm 0.03 \mathrm{~L} \cdot \mathrm{min}^{-1} \cdot \mathrm{m}^{-2}$. For $\mathrm{mPAD}$, the error was $1.33 \pm 5.70 \%$. The Bland-Altman analysis for agreement between the two techniques for CI measurements (RHC versus MRI) showed an error of $0.33 \pm 0.69 \mathrm{~L} \cdot \mathrm{min}^{-1} \cdot \mathrm{m}^{-2}$ (fig. 2).

The mPAD differed significantly in responders and nonresponders, as shown in figure $3(p=0.01)$. Based on the ROC curve, a cut-off value of $10 \% \mathrm{mPAD}$ was chosen arbitrarily in order to differentiate between responders and nonresponders with $100 \%$ sensitivity (95\% confidence interval of $61-100 \%$ ) and $56 \%$ specificity (33-77\%). A positive predictive value of $36 \%(15-65 \%)$ and a negative predictive value of $100 \%$ (77$100 \%$ ) were also found.

No significant relationship has been found between mPAD and $\bar{P}_{\text {pa }}(\mathrm{r}=-0.25, \mathrm{p}=0.28)$, and PVRi $(\mathrm{r}=0.02, \mathrm{p}=0.91)$ and $\mathrm{CI}$ $(\mathrm{r}=-0.14, \mathrm{p}=0.54)$. In two patients, the image quality obtained was not sufficient to allow a proper estimation of ventricular volumes and masses. However, a significant correlation between RAP and right ventricle ejection fraction (RVEF) was found $(\mathrm{n}=17, \mathrm{r}=-0.60,95 \%$ confidence interval $0.17-0.84$; $\mathrm{p}<0.01$ ), as well as between $P$ pao and left ventricle ejection fraction $(\mathrm{LVEF})(\mathrm{n}=17, \mathrm{r}=-0.59,95 \%$ confidence interval 0.18 $0.83 ; \mathrm{p}=0.01)$.

EF from LV and RV, assessed using MRI, have also shown significant correlation $(\mathrm{n}=17, \mathrm{r}=0.51,95 \%$ confidence interval 0.03-0.80; $\mathrm{p}<0.05)$. Correlation between RV mass/LV mass ratio and $\bar{P}_{\text {pa }}$ was significant as well $(n=17, r=-0.62,95 \%$ confidence interval $0.20-0.85 ; \mathrm{p}<0.05$ ).

No statistically significant correlation was observed between the variation of $\bar{P}_{\text {pa }}\left(\bar{P}_{\text {pa }}\right.$ before $/ \bar{P}_{\text {pa after }}$ NO) and mPAD ( $\mathrm{n}=19, \mathrm{r}=0.37 ; \mathrm{p}=$ nonsignificant), and between $\mathrm{mPAD}$ and $\bar{P}_{\mathrm{pa}}$ at rest $(n=19, r=-0.25 ; p=$ nonsignificant $)$. 


\begin{tabular}{|c|c|c|c|c|}
\hline \multirow[t]{3}{*}{ TABLE 2} & $\begin{array}{l}\text { nodynamic } \\
\text { (NO) testir }\end{array}$ & $\begin{array}{l}\text { data accorc } \\
\text { ig }\end{array}$ & ding to acut & te nitric \\
\hline & \multicolumn{2}{|c|}{ Responders $^{\#, \text {, }}$} & \multicolumn{2}{|c|}{ Nonresponders ${ }^{\#,+}$} \\
\hline & Baseline & NO & Baseline & NO \\
\hline $\bar{P}$ pa $\mathbf{m m H g}$ & $60 \pm 6$ & $31 \pm 8$ & $72 \pm 23$ & $63 \pm 21$ \\
\hline PVRi dyn $\cdot \mathbf{s} \cdot \mathbf{c m}^{-5} \cdot \mathbf{m}^{-2}$ & $2314 \pm 919$ & $1219 \pm 1172$ & $2311 \pm 1793$ & $1840 \pm 1126$ \\
\hline CI L. $\min ^{-1} \cdot \mathrm{m}^{-2}$ & $2.04 \pm 0.66$ & $2.88 \pm 0.68$ & $2.47 \pm 1.14$ & $2.45 \pm 1.08$ \\
\hline Ppao mmHg & $10 \pm 2$ & $9 \pm 1$ & $8 \pm 2$ & $9 \pm 1$ \\
\hline RAP $\mathbf{m m H g}$ & $7 \pm 6$ & $5 \pm 4$ & $13 \pm 6$ & $12 \pm 6$ \\
\hline \multicolumn{5}{|c|}{$\begin{array}{l}\text { Data are presented as mean } \pm \text { SD. } \bar{P} \text { pa: mean pulmonary artery pressure; PVRi: } \\
\text { indexed pulmonary vascular resistance; Cl: cardiac index; Ppao: pulmonary } \\
\text { artery occlusion pressure; RAP: right atrial pressure. \#: no statistically } \\
\text { significant differences were observed in baseline haemodynamics between } \\
\text { responders and nonresponders; }{ }^{\ominus}: n=4 ;^{+}: n=15 \text {. }\end{array}$} \\
\hline
\end{tabular}

\section{DISCUSSION}

The present results show that there is a significant difference in the mPAD of responders and nonresponders. Consequently, this parameter could be used as a tool to differentiate these two groups of patients through a baseline MRI.

Distensibility measurements between the two observers showed excellent agreement. Previous MRI studies for anatomical investigation have found similar results [21]. The mPAD was significantly higher in acute $\mathrm{NO}$ responders in comparison with nonresponders $(27.3 \pm 18.4$ and $11.0 \pm 6.8 \%$, respectively; $\mathrm{p}<0.05)$. Interesting data are available on the literature regarding right pulmonary artery distensibility (PAD) and its relation to pulmonary artery pressure [13]. However, to the best of the present authors' knowledge, no other study has evaluated this parameter as a noninvasive predictor of acute NO response. There are known differences in $\mathrm{mPAD}$ in $\mathrm{PAH}$ patients when compared with normal individuals, as stated by BOGREN et al. [19] (mPAD was $8 \%$ in PAH patients) and CASALINO et al. [21] (mPAD in HIVassociated pulmonary hypertension patients was $18 \%$ versus $26 \%$ in the control group; $\mathrm{p}<0.05$ ). In normal individuals the average mPAD was reported to be $\sim 25 \%$ [24]. The fact that $\mathrm{mPAD}$ in responders is similar to $\mathrm{MPAD}$ in normal individuals of other studies suggests that elevation in $\bar{P}_{\mathrm{pa}}$ is not the main factor involved in MPAD reduction.

This hypothesis was addressed in the present study and no correlation was found between $\bar{P}$ pa and mPAD. The correlation between variation in $\bar{P}_{\text {pa }}\left(\bar{P}_{\text {pa }}\right.$ before $/ \bar{P}_{\text {pa }}$ during NO) and $\mathrm{mPAD}$ has also been tested to see whether pressure variation brings a variation in distensibility. Again, no significant correlation was found. Pulmonary circulation assessment through analysis of proximal vessels may have limitations and is a matter of debate. It is known that there is an inverse correlation between pulmonary artery compliance and pressure levels [25]. However, when $\bar{P}_{\text {pa }}$ reaches $40 \mathrm{mmHg}$, this relationship tends to flatten and become stable [26] and thus the proximal part of the system would better reflect the behaviour of the distal arteries, particularly in $\mathrm{PAH}$ where changes in elastic arteries lead to a diminished compliance of

\section{TABLE 3 Functional data}

\begin{tabular}{|c|c|}
\hline Subjects n & 19 \\
\hline CI L. $\min ^{-1} \cdot \mathrm{m}^{-2}$ & $1.98 \pm 0.71$ \\
\hline LVEF $^{\#} \%$ & $58 \pm 13$ \\
\hline RVEF $^{\#} \%$ & $32 \pm 16$ \\
\hline Left ventricular mass ${ }^{\#} \mathbf{g}$ & $75 \pm 25$ \\
\hline Right ventricular mass $^{\#} \mathbf{g}$ & $42 \pm 19$ \\
\hline LV end diastolic volume $\mathrm{mL}$ & $69 \pm 24$ \\
\hline $\mathrm{RV}$ end diastolic volume $\mathrm{mL}$ & $108 \pm 41$ \\
\hline Pulmonary artery SA mm² & $1407 \pm 600$ \\
\hline Pulmonary artery DA mm² & $1259 \pm 605$ \\
\hline mPAD \% & $14 \pm 11$ \\
\hline
\end{tabular}

Data presented as mean $\pm \mathrm{SD}$, unless otherwise stated. Cl: cardiac index; LVEF: left ventricle ejection fraction; RVEF: right ventricle ejection fraction; SA: systolic area; DA: diastolic area; mPAD: mean pulmonary artery distensibility. ${ }^{\#}: \mathrm{n}=17$, as in two patients the estimation of ventricular volumes and masses was not performed.

the whole vascular system $[26,27]$. Taking these factors and the present findings into consideration, the mPAD may be a marker of pulmonary vascular remodelling processes and not a consequence of the haemodynamic pattern. Although interesting, this hypothesis would require the analysis of pathology lung specimens to be confirmed.

In order to further address MPAD use in the clinical setting, determination of the value that would differentiate acute NO responders from nonresponders according to ROC curve analysis was undertaken. The area under the curve was found to be $0.83(\mathrm{p}<0.05)$ and a value of $10 \%$ mPAD was arbitrarily chosen as a cut-off point. The decision to select this cut-off value, which determines high sensitivity and low specificity, was based on the fact that recognising the larger population of patients who should not be treated with CCBs is more important than accurately recognising the small proportion of responders. Thus, the use of a cut-off value with high sensitivity will avoid the need for a significant number of acute

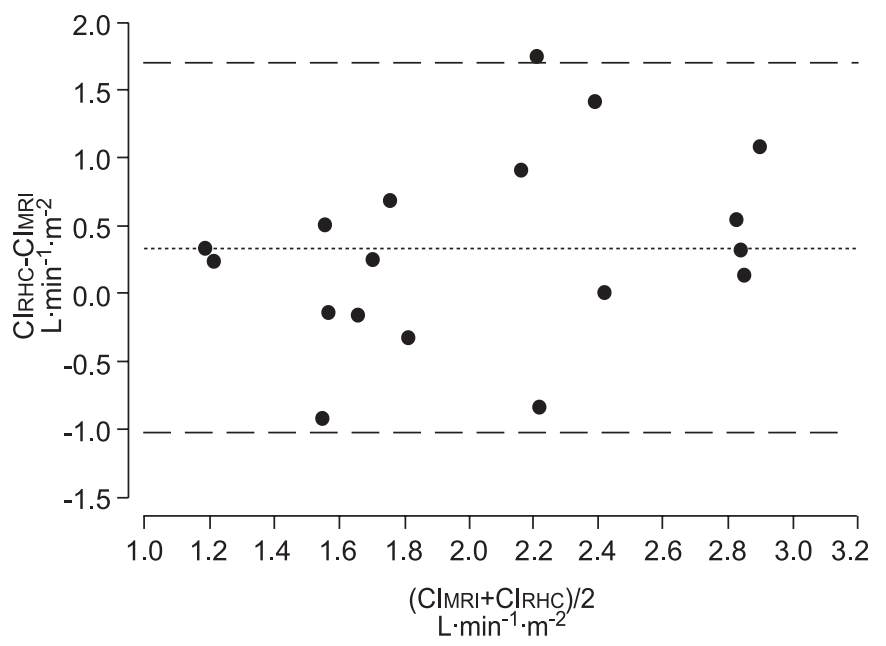

FIGURE 2. Bland-Altman analysis of cardiac index (Cl) estimated using magnetic resonance imaging (CIMRI) and right heart catheterisation (ClRHC). …... mean; -----: mean $\pm 1.96 \mathrm{sD}$ 


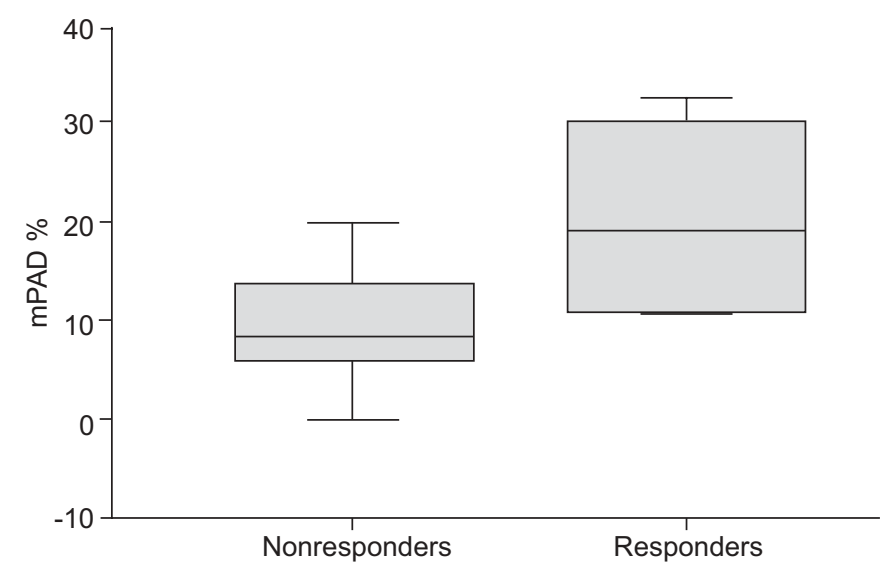

FIGURE 3. Mean pulmonary artery distensibility (mPAD) in responders and nonresponders $(p=0.01)$.

vasodilator tests at baseline. This could be particularly important when considering patients who have already undergone RHC prior to being referred to a pulmonary hypertension centre.

The high negative predictive value of $\mathrm{MPAD}$, considering this cut-off value for the differentiation between responders and nonresponders, brings up the prospect of using of mPAD as a noninvasive marker of acute response. This might be of interest not only for ruling out the use of CCBs in nonresponders but also as a follow-up tool in the evaluation of the cardiovascular system in the setting of the available therapies for PAH treatment. With the development of the $64 \mathrm{CT}$ detector, gatedCT images might also enable the estimation of PAD at the screening for thromboembolic disease during $\mathrm{PAH}$ investigation. However, this hypothesis should be addressed in prospective studies comparing MRI and CT images.

The present results, however, should be scrutinised considering the small number of patients enrolled in the current pilot study. Even though the clinical and haemodynamic characteristics of these patients were similar to the previously described data from IPAH patients [28] and baseline haemodynamic data did not differ significantly between responders and nonresponders, the proportion of responders is higher than previously described for the defined response criteria [8].

Although not the primary objective of the present study, the use of MRI also allowed the noninvasive estimation of haemodynamic data, such as the CI. The interobserver agreement for the estimation of CI was excellent, probably due to the fact that the CI was calculated from aorta flow measurements and, since the aorta is an extremely regular vessel, there is little room for involuntary measurement misjudgement at the semi-automatic contour detection used for the flow analysis [19, 21, 24].

The mean error found when analysing the agreement between CI measured using MRI and RHC shows that, although MRI seems to be an interesting noninvasive method for haemodynamic assessment [16], CIMRI cannot be used as a substitute for CIRHC at the present time. Nevertheless, the potential use of CIMRI as a follow-up tool in order to demonstrate the trend towards improvement or deterioration in CI during treatment remains to be addressed.

The limited number of patients studied did certainly influence the magnitude of the mean error between the two methods. Moreover, RHC using thermodilution for CI determination may not be the ideal gold standard, as many factors, including tricuspid regurgitation, affect the accuracy of $\mathrm{CI}$ measurements [7]. Future studies should compare cardiac output measurements using thermodilution, MRI and the Fick method.

Other data obtained using MRI image analysis also set interesting perspectives for its use. Significant correlations between RAP and RVEF, as well as Ppao and LVEF, show the relationships between ventricular pumps and upstream pressures, emphasising the potential ability of a noninvasive technique, such as MRI, in reflecting important prognostic markers in PAH patients, such as RAP.

Another interesting finding was the significant correlation between RVEF and LVEF. Previous studies in PAH patients have shown relationships between RV and LV volumes [18] and interventricular interdependence [29]. The present findings regarding right and left ventricular function relationships are believed to reinforce the pathophysiological findings previously reported.

In conclusion, the present pilot prospective monocentre study indicates that noninvasive assessment of pulmonary artery distensibility using magnetic resonance imaging reflects the acute response pattern in idiopathic pulmonary arterial hypertension patients. These encouraging results, together with the prospect of using magnetic resonance imaging for haemodynamic measurements, should be confirmed in a larger prospective multicentre study.

\section{REFERENCES}

1 Fishman AP. Primary pulmonary arterial hypertension: a look back. J Am Coll Cardiol 2004; 43: Suppl. 12, 2S-4S.

2 Dawson CA, Krenz GS, Karau KL, Haworth ST, Hanger CC, Linehan JH. Structure-function relationships in the pulmonary arterial tree. J Appl Physiol 1999; 86: 569-583.

3 Farber HW, Loscalzo J. Pulmonary arterial hypertension. N Engl J Med 2004; 351: 1655-1665.

4 Barst RJ, McGoon M, Torbicki A, et al. Diagnosis and differential assessment of pulmonary arterial hypertension. J Am Coll Cardiol 2004; 43: Suppl. 12, 40S-47S.

5 Simonneau G, Galie N, Rubin LJ, et al. Clinical classification of pulmonary hypertension. J Am Coll Cardiol 2004; 43: Suppl. 12, 5S-12S.

6 Humbert M, Sitbon O, Simonneau G. Treatment of pulmonary arterial hypertension. N Engl J Med 2004; 351: 1425-1436.

7 Chemla D, Castelain V, Hervé P, Lecarpentier Y, Brimioulle S. Haemodynamic evaluation of pulmonary hypertension. Eur Respir J 2002; 20: 1314-1331.

8 Sitbon O, Humbert M, Jais X, et al. Long-term response to calcium channel blockers in idiopathic pulmonary arterial hypertension. Circulation 2005; 111: 3105-3111. 
9 Sitbon O, Brenot F, Denjean A, et al. Inhaled nitric oxide as a screening vasodilator agent in primary pulmonary hypertension. A dose-response study and comparison with prostacyclin. Am J Respir Crit Care Med 1995; 151: 384-389.

10 Sitbon O, Humbert M, Jagot JL, et al. Inhaled nitric oxide as a screening agent for safely identifying responders to oral calcium-channel blockers in primary pulmonary hypertension. Eur Respir J 1998; 12: 265-270.

11 Costa EL, Jardim C, Bogossian HB, Amato MB, Carvalho CR, Souza R. Acute vasodilator test in pulmonary arterial hypertension: evaluation of two response criteria. Vascul Pharmacol 2005; 43: 143-147.

12 Souza R, Bogossian HB, Humbert M, et al. N-terminal-probrain natriuretic peptide as a haemodynamic marker in idiopathic pulmonary arterial hypertension. Eur Respir J 2005; 25: 509-513.

13 Vonk-Noordegraaf A, Marcus JT, Holverda S, Roseboom B, Postmus PE. Early changes of cardiac structure and function in COPD patients with mild hypoxemia. Chest 2005; 127: 1898-1903.

14 Bossone E, Bodini BD, Mazza A, Allegra L. Pulmonary arterial hypertension: the key role of echocardiography. Chest 2005; 127: 1836-1843.

15 Schoepf UJ, Kucher N, Kipfmueller F, Quiroz R, Costello P, Goldhaber SZ. Right ventricular enlargement on chest computed tomography: a predictor of early death in acute pulmonary embolism. Circulation 2004; 110: 3276-3280.

16 Saba TS, Foster J, Cockburn M, Cowan M, Peacock AJ. Ventricular mass index using magnetic resonance imaging accurately estimates pulmonary artery pressure. Eur Respir J 2002; 20: 1519-1524.

17 Mousseaux E, Tasu JP, Jolivet O, Simonneau G, Bittoun J, Gaux JC. Pulmonary arterial resistance: noninvasive measurement with indexes of pulmonary flow estimated at velocity-encoded MR imaging-preliminary experience. Radiology 1999; 212: 896-902.

18 Boxt LM, Katz J, Kolb T, Czegledy FP, Barst RJ. Direct quantitation of right and left ventricular volumes with nuclear magnetic resonance imaging in patients with primary pulmonary hypertension. J Am Coll Cardiol 1992; 19: 1508-1515.

19 Bogren HG, Klipstein RH, Mohiaddin RH, et al. Pulmonary artery distensibility and blood flow patterns: a magnetic resonance study of normal subjects and of patients with pulmonary arterial hypertension. Am Heart J 1989; 118: 990-999.

20 Berger RM, Cromme-Dijkhuis AH, Hop WC, Kruit MN, Hess J. Pulmonary arterial wall distensibility assessed by intravascular ultrasound in children with congenital heart disease: an indicator for pulmonary vascular disease? Chest 2002; 122: 549-557.

21 Casalino E, Laissy JP, Soyer P, Bouvet E, Vachon F. Assessment of right ventricle function and pulmonary artery circulation by cine-MRI in patients with AIDS. Chest 1996; 110: 1243-1247.

22 Vincent JL. What is right ventricular (dys)function? Crit Care Med 1994; 22: 2024-2026.

23 Saito H, Dambara T, Aiba M, Suzuki T, Kira S. Evaluation of cor pulmonale on a modified short-axis section of the heart by magnetic resonance imaging. Am Rev Respir Dis 1992; 146: 1576-1581.

24 Paz R, Mohiaddin RH, Longmore DB. Magnetic resonance assessment of the pulmonary arterial trunk anatomy, flow, pulsatility and distensibility. Eur Heart J 1993; 14: 1524-1530.

25 Harris P, Heath D, Apostolopoulos A. Extensibility of the human pulmonary trunk. Br Heart J 1965; 27: 651-659.

26 Reuben SR. Compliance of the human pulmonary arterial system in disease. Circ Res 1971; 29: 40-50.

27 Reeves JT, Linehan JH, Stenmark KR. Distensibility of the normal human lung circulation during exercise. Am J Physiol Lung Cell Mol Physiol 2005; 288: L419-L425.

28 Rich S, Dantzker DR, Ayres SM, et al. Primary pulmonary hypertension. A national prospective study. Ann Intern Med 1987; 107: 216-223.

29 Pinsky MR. Recent advances in the clinical application of heart-lung interactions. Curr Opin Crit Care 2002; 8: 26-31. 